\title{
Realization of Virtual-Link on a Network
}

\author{
Anil Umesh ${ }^{1}$ \\ BE, Department of CSE, SJCE, Mysore, India ${ }^{1}$
}

\begin{abstract}
A network is group of computers and other devices running on suitable protocols enabling data and multimedia communication. Multimedia includes image, audio, video and text. Router is a part of the network and is responsible for forwarding the packets. A routing protocol specifies how routers communicate with each other. Open Shortest Path First (OSPF) is a dynamic routing protocol and uses Dijkstra shortest path first algorithm to determine the shortest path. OSPF employs a hierarchical network design using areas. Area 0 is the backbone area. All other areas that is area $n$, where $n=1,2,3 \ldots$ are (must be) directly connected to the backbone. But in several cases the nonbackbone area cannot be directly connected to area 0 . In this paper we present to you the configuration of virtual-links to logically connect separated areas to area 0 .
\end{abstract}

Keywords: Data Communication, Multimedia Communication, Open Shortest Path First (OSPF), Hierarchical Network Design, Virtual-Links to Logically Connect Separated Areas to Area 0, Autonomous Systems (AS)

\section{INTRODUCTION}

Open Shortest Path First protocol is an intra-domain routing protocol based on link state routing. OSPF protocol supports area to handle routing efficiently and in a timely manner. An autonomous system can be divided into many areas. All networks inside an area must be connected. Area 0 is called the backbone area. Area $n$ where $n=1,2,3 \ldots$. are called non-backbone areas. An area is a collection of networks, hosts, routers and other network components. All nonbackbone areas must be connected to the backbone area. The router between two areas is called an Area Border Router (ABR). A router between two Autonomous Systems (AS) is called an Autonomous System Border Router (ASBR). Figure 1 shows the hierarchical network design of an OSPF network with area 0 as the backbone area.

\section{FEASIBILITY STUDY}

Feasibility is a signified process carried out over various aspects of a commercial project. In the realization of virtuallink, GNS 3 tool is used to simulate the network and check for connectivity. The simulated routers have been observed to exchange data with the other routers without any discrepancies. Hierarchical network consisting of areas with unique area identification number could be flooded with LSA packets. [1]

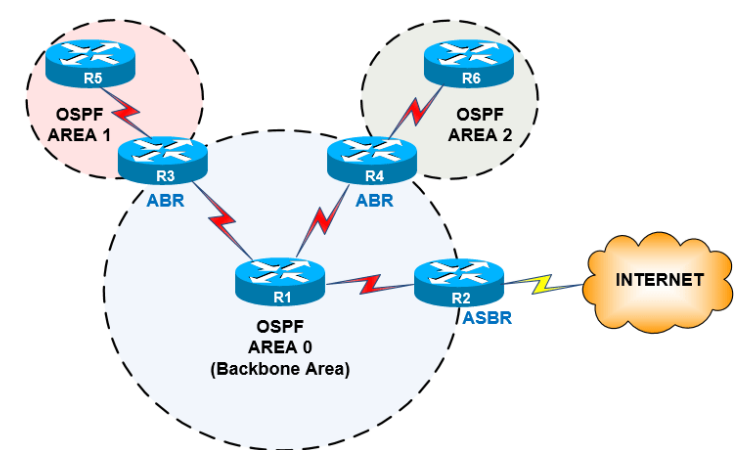

Figure 1 shows the hierarchical network design of an OSPF network with area 0 as the backbone area.

\section{SOFTWARE REQUIREMENTS SPECIFICATION}

A. Functional Requirements

Functional requirements define the functionality of a system to be developed. It also reflects each functionality of the user in the system. The functional requirements of the proposed system are

- $\quad$ Selection of proper routers and other networks best suited for the situation.

- $\quad$ Providing proper addressing scheme for the packets to traverse through the network.

- Use of proper dynamic or static routing protocols to guide the packets in the network.

- $\quad$ Proper OSPF area identification scheme. 


\section{International Journal of Advanced Research in Computer and Communication Engineering}

Vol. 7, Issue 9, September 2018

B. Non-functional Requirements

Non-functional requirements play the behaviour and performance of the system at its critical stages

- Scalability

Provisions will be made to accommodate more routers or network elements to avoid early saturation in the network.

- $\quad$ Security

The system will provide secured transfer of data between nodes within the AS

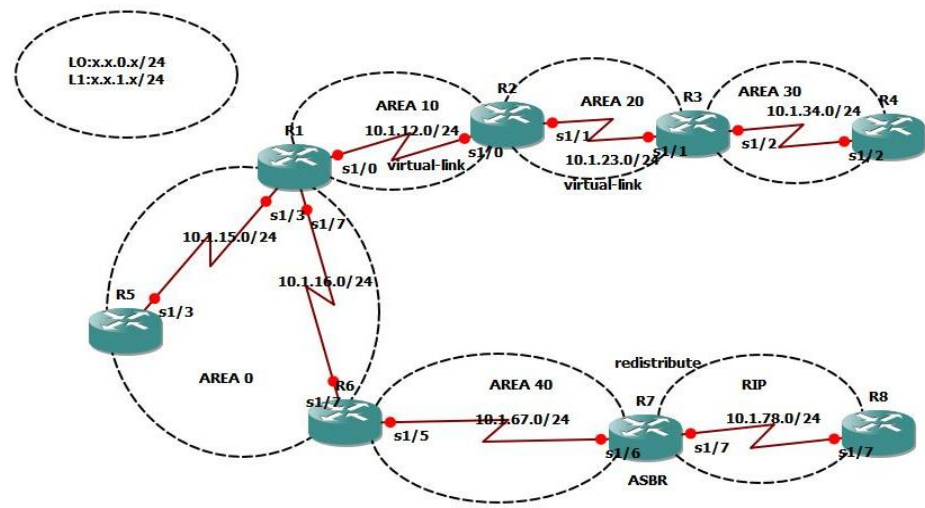

Figure 2 shows virtual-link, a tunnel between area 20 and area 0.

\section{PROBLEM STATEMENT AND SOLUTION}

OSPF is a hierarchical system that separates an Autonomous System (AS) into individual areas. It consists of [2]

- $\quad$ Backbone area= area 0 .

- $\quad$ Non backbone areas $=$ area $n$, where $n=1,2,3$.. and $n !=0$.

- Transient area= which is a non-backbone area.

All areas must be connected to the backbone area or area 0. In normal OSPF operations this may not be possible. There are circumstances preventing direct connection of other areas to area 0. A general solution to this problem is the configuration of virtual-link between the areas [3], logically connecting separated areas to area 0 . Figure 2 shows virtual-link, a tunnel between area 20 and area 0.

\section{SIMULATION AND RESULTS}

In this paper efforts were put in to build a network and configure OSPF on it. OSPF network being a hierarchical network consists of areas and virtual-links. Figure 3 and 4 shows the configuration of virtual-link. Figure 5 shows the results when $\mathrm{R} 1$ tried to ping $\mathrm{R} 3$. The success rate was $100 \%$, as all the 5 ICMP echo packets returned with a TTL value of 2 seconds.

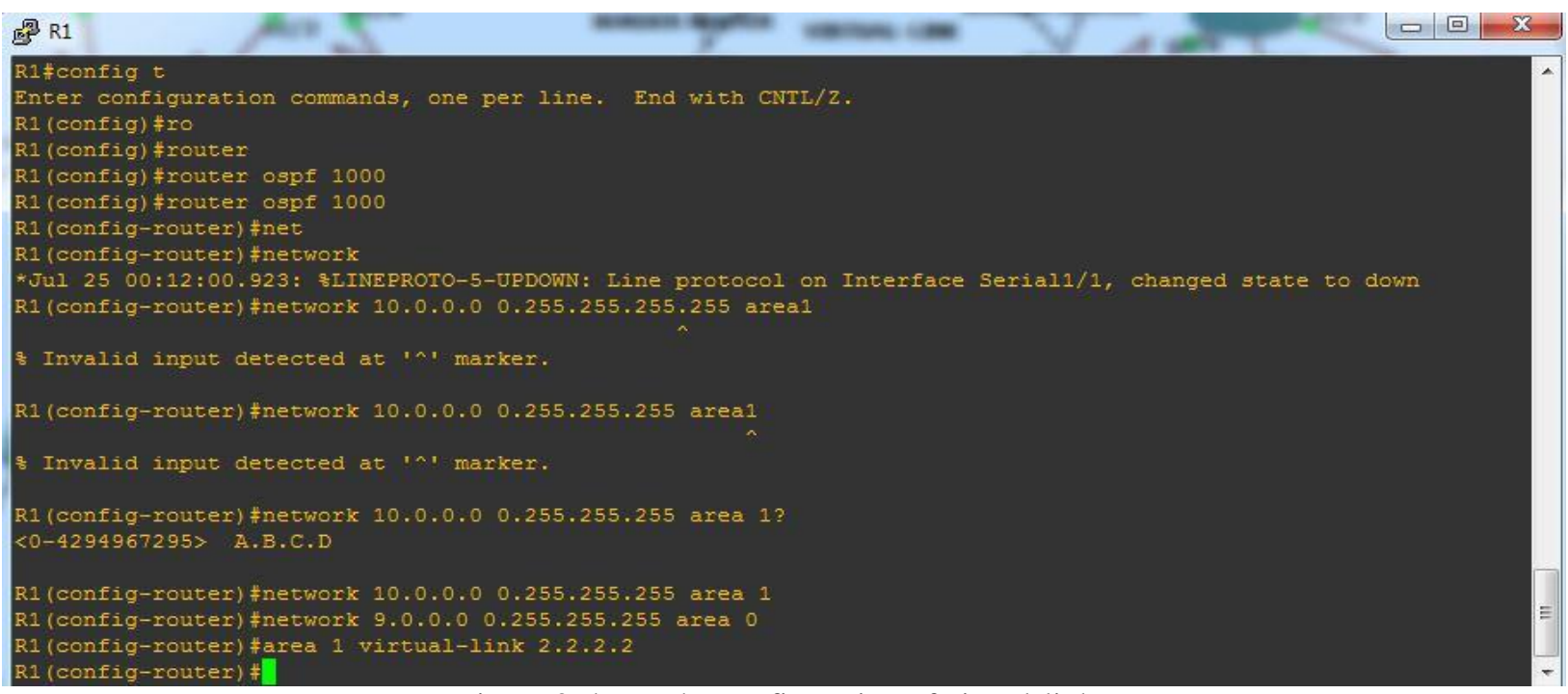

Figure 3 shows the configuration of virtual-link. 


\section{International Journal of Advanced Research in Computer and Communication Engineering}

Vol. 7, Issue 9, September 2018

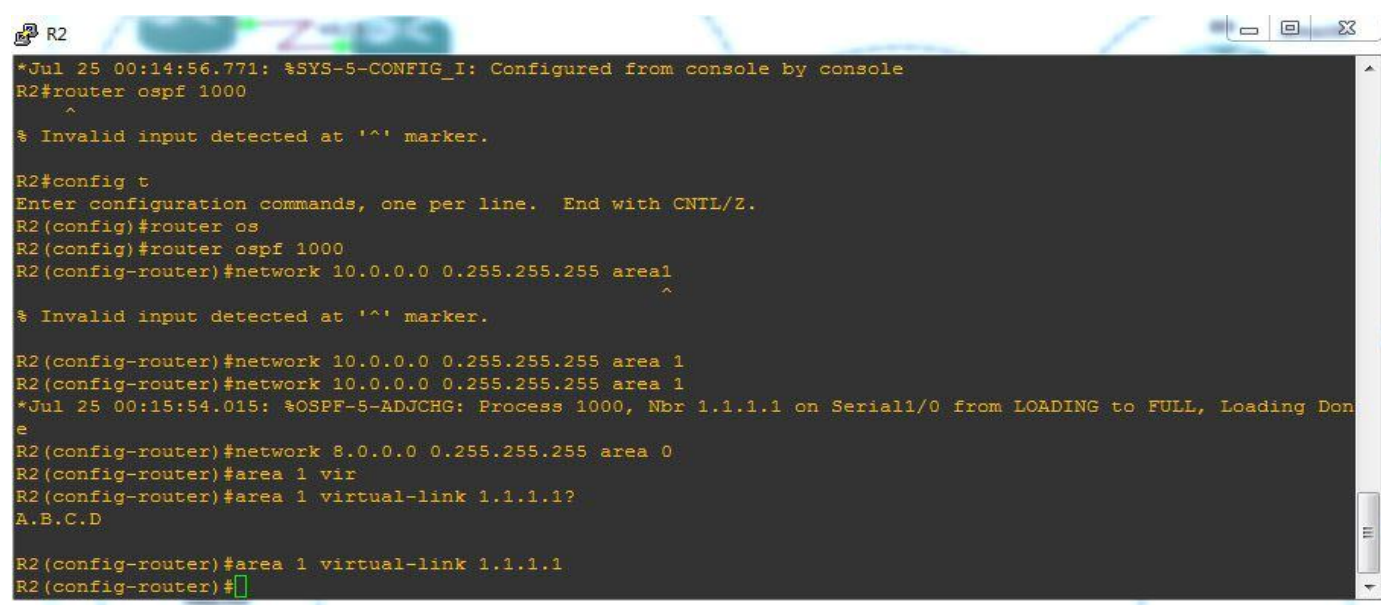

Figure 4 shows the configuration of virtual-link.

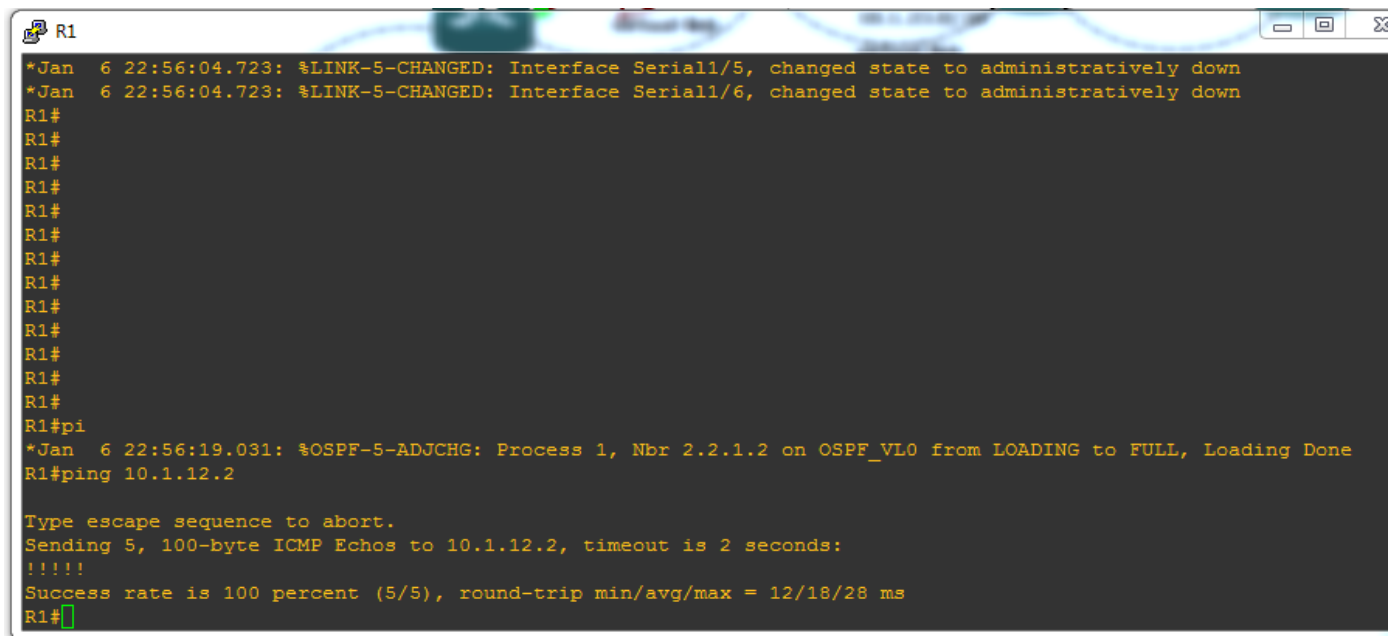

Figure 5 shows the results when R1 tried to ping R3.

\section{CONCLUSIONS}

Open Shortest Path First (OSPF) is a dynamic routing protocol and employs a hierarchical network design using areas. Any OSPF network consists of a backbone area, called area 0 . All other areas must be connected to the backbone. It so happens in reality that, the non-backbone areas cannot be directly connected to the backbone area. A virtual-link is a logical connection and acts as a tunnel between separated areas and area 0 . In this paper virtual-link was successfully configured on the OSPF network and results published.

\section{REFERENCES}

[1]. Securing the Internet Routing Infrastructure- Panagiotis Papadimitratos and Zygmunt J. Haas, Cornell University.

[2]. To Minimize the Consumption of Logical Addresses in a Network using OSPF with Overloading Technique- by Neha Grang \& Anuj K.Gupta

[3]. OSPF virtual-links- https://www.cisco.com/c/en/us/support/docs/ip/open-shortest-path-first-ospf/47866-ospfdb7.pdf

\section{OUR GUIDE}

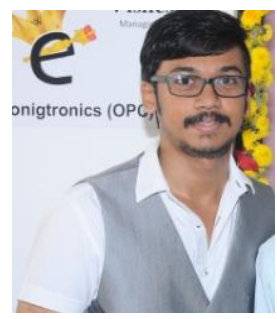

VISHESH S born on $13^{\text {th }}$ June 1992, hails from Bangalore (Karnataka) and has completed B.E in Telecommunication Engineering from VTU, Belgaum, Karnataka in 2015. He also worked as an intern under Dr. Shivananju BN, former Research Scholar, Department of Instrumentation, IISc, Bangalore. His research interests include Embedded Systems, Wireless Communication, BAN and Medical Electronics. He is also the Founder and Managing Director of the corporate company Konigtronics Private Limited. He has guided over a hundred students/interns/professionals in their research work and projects. He is also the co-author of many International Research Papers. He is currently pursuing his MBA in e-Business and PG Diploma in International Business. Presently Konigtronics Private Limited has extended its services in the field of Software Engineering and Webpage Designing. Konigtronics also conducts technical and non-technical workshops on various topics. 